\title{
Situation Awareness and Early Recognition of Traffic Maneuvers
}

\author{
Galia Weidl $^{1} \quad$ Anders L. Madsen $^{2,4} \quad$ Viacheslav Tereshchenko $^{1,3}$ \\ Wei Zhang ${ }^{1,3}$ Stevens Wang ${ }^{1,3}$ Dietmar Kasper ${ }^{1}$ \\ ${ }^{1}$ Department of Driving Automation, Daimler AG, Group Research \& AE, 71034 Böblingen, Germany \\ \{galia.weidl, dietmar.kasper\} edaimler.com \\ ${ }^{2}$ HUGIN EXPERT A/S, ${ }^{4}$ Department of Computer Science, Aalborg University, Denmark \\ anders@hugin.com \\ ${ }^{3}$ University of Stuttgart, Germany \\ \{viacheslav.tereshchenko, zw158535165, stevens.rx.wang\}@gmail.com
}

\begin{abstract}
We outline the challenges of situation awareness with early and accurate recognition of traffic maneuvers and how to assess them. This includes also an overview of the available data and derived situation features, handling of data uncertainties, modelling and the approach for maneuver recognition. An efficient and effective solution, meeting the automotive requirements, is successfully deployed and tested on a prototype car. Test driving results show that earlier recognition of intended maneuver is feasible on average 1 second (and up to $6.72 \mathrm{~s}$ ) before the actual lane marking crossing. The even earlier maneuver recognition is dependent on the earlier recognition of surrounding vehicles.
\end{abstract}

Keywords: Bayesian networks, massive data streams

\section{Introduction}

A highway, typically providing several traffic lanes, is characterized by complex scenes with many vehicles. Reliable situation assessment requires multi-sensor fusion and management of uncertainty in order to interpret accurately the traffic environment. To reduce the risk of accidents and congestions, an autonomous system must analyze and be aware of possible hazards of a driving situation. This includes: correctly recognizing intended maneuvers of all surrounding vehicles at an early stage and using this information to enable corrective actions like braking or steering, thus helping to avoid or mitigate potential collisions. Situational awareness and recognition of traffic maneuvers are key elements of modern driver assistance and autonomous driving systems (Kasper et al, 2011, 2012, 2013; Morris et al, 2011; Kumar et al, 2013; Tereshchenko, 2014; Schlechtriemen et al, 2014; Weidl et al, 2014, 2015, 2017; Mori et al, 2015; Satzoda et al, 2015; Zeisler et al, 2015).

A probabilistic approach, using Object-Oriented Bayesian networks (OOBNs) for maneuver recognition has been proposed in (Kaper et al, 2011, 2012, 2013) and (Zeisler et al, 2015). It is based on the own (ego) vehicle dynamics, its driving path in relations to the lane markings and/or surrounding vehicles, to evaluate the vehicles' relevance as possible target objects and to recognize earlier maneuvers in real traffic. In addition to the data from in-vehicle sensors, including both the vehicle kinematics and vehicle surround dynamics, (Satzoda et al, 2015) also uses visual data from multiple perspectives to characterize lane changes. In (Kaper et al, 2011, 2012, 2013), we use pairwise vehicle-vehicle relations; as far as the sensors can percept the surrounding objects.

In (Mori et al, 2015) an approach, based on Hidden Markov Models computes the driver's intent on lane change and/or potential risk of accidents. This work studied the driver decisions whether is safer to change lane in front of a faster car closing the gap or to brake for keeping a safe distance to a slower car in front, by evaluating the time-to-collision (TTC). We have used similar features in the safety model (in section 3 ) to infer the intention on lane change for the situations shown in Figure 1, utilizing the relative longitudinal dynamic between a vehicle (own, neighbor) and the vehicles in front and back, driving on the target lane (Tereshchenko, 2014). This effectively builds a gap for finishing a lane change maneuver and has an impact on the decision for lane changing, see (Tereshchenko, 2014; Mori et al, 2015; Weidl et al, 2015; Yan et al, 2015). The mentioned features, characterizing the vehicle state, were extended in ( $\mathrm{Li}$ et al, 2015) with the driver's operation signals to enhance by hidden Markov models the classification of lane change maneuvers.

In our recent work (Weidl et al, 2015) and for the results reported here, we have focused on the development of a solution for maneuver recognition and its deployment on a Linux based system emulating the automotive target platform, using commercially viable sensors, image processing and multi-sensor fusion. We use the commercial software HUGIN, allowing efficient $\mathrm{BN}$ modelling and automated c-code generation. All 
current and future developments are compared to our initially developed "Original (ORIG)" OOBN, which has shown promising results as described in (Kasper et al, 2011, 2012, 2013; Weidl et al, 2014). Our latest work (Weidl et al, 2015) describes three statistical classifiers as deployed on the prototype vehicle as well as the planned dynamic extension into a Dynamic Bayesian Network (DBN). The DBN are now deployed on the vehicle and evaluated in highway drive and in statistical comparison to other static classifiers. The main contribution of this work is the extension of all four classifiers with special evidence for better accuracy. The DBN deployment on the automotive target platform has been successfully tested in real highway driving.

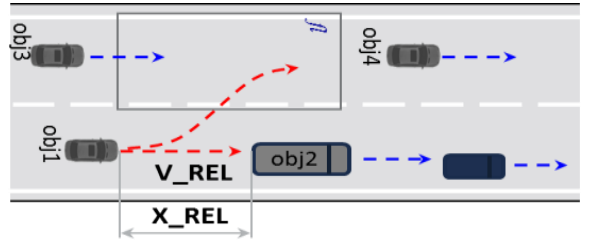

Figure 1. Longitudinal relative dynamics between following and front vehicles, both moving on the same lane. Lateral relative dynamics towards the lane marking, when initiating a lane change maneuver.
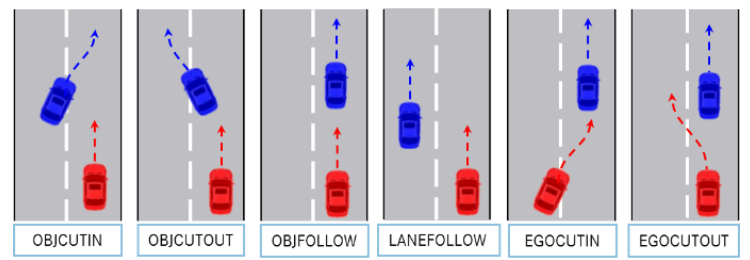

Figure 2. Maneuver recognition as a vehicle-vehicle relation between the own EGO-vehicle (red) and a neighbor OBJ-vehicle (blue).

The automotive target platform represents both a storage and an inference challenge. The requirement on automotive safety demands accuracy close to $100 \%$ for a prediction horizon of 1 second. Moreover, the solution should scale to the specification of the hardware restrictions of the target platform. It should meet the automotive requirements on computation time and memory space, which are strongly constrained by the electronic control unit. The quick development of situations over time requires an automatic system, capturing and analyzing massive data streams, under uncertainties, every 20 milliseconds for several safety applications, resulting in 0.15 milliseconds for maneuver recognition.

This paper is organized as follows. Section 2 gives an overview of the used method, section 3 - on efficient modeling, section 4 - on proper treatment of data uncertainties and their use for building of hypotheses on driving behavior for event (maneuver) recognition. The approach is outlined in section 5, while section 6 describes the evaluation of classifiers. Section 7 summarizes the results with outlook.

\section{Method for Probabilistic Reasoning}

\subsection{Bayesian network (BN)}

A Bayesian network $\boldsymbol{B N}:=(\boldsymbol{G}, \boldsymbol{P})$ is defined as a directed acyclic graph $\boldsymbol{G}$ and $\boldsymbol{P}$ - a set of CPDs (conditional probability distributions) $\boldsymbol{P}(\boldsymbol{X} \mid \boldsymbol{p a}(\boldsymbol{X}))$ of a variable $X$ conditioned on its influence variables $p a(X)$, (Friedman and Koller 2009, Kjærulff and Madsen 2013). The joint probability of a $\mathrm{BN}$ is computed by the Chain rule for BNs:

$$
P\left(X_{1}, \ldots, X_{n}\right)=\Pi_{i=1 . . n} P\left(X_{i} \mid p a\left(X_{i}\right)\right)
$$

The graph $G=(V, L)$ contains nodes $\mathrm{V}$ (to represent random variables) and edges $\mathrm{L}$ to represent the conditional dependency relations between the nodes. A BN can be used as a knowledge representation to compute the probability $\boldsymbol{P}(\boldsymbol{X}=\boldsymbol{x} \mid \boldsymbol{e})$ given a set of observations $e$. The Bayesian theorem allows inverting the probability computations, i.e.

$$
P(X \mid Y)=P(Y \mid X) P(X) / P(Y)
$$

An object-oriented Bayesian network (OOBN) contains instance nodes in addition to the usual BN nodes. An instance node is an abstraction of a net fragment into a single unit (network class) (Friedman and Koller 2009, Kjærulff and Madsen 2013). Therefore, instance nodes can be used to represent different network classes as well as repetitive structures within other nets (encapsulation). Thus an OOBN can be viewed as a hierarchical (data/information fusion) model of a problem domain. Every layer in this hierarchy expresses another level of abstraction in the OOBN model. The modeling extensions in this work explore also dynamic Bayesian networks (DBN), which use a time series of observations for information fusion and inference (Friedman and Koller 2009; Zhang and Ji 2009; Kjærulff and Madsen 2013; Weidl et al, 20152017). In this work, we use OOBN and DBN to represent the extension for both the lateral and longitudinal relative dynamics. DBN combine repetitive BN structures as discrete time slices. They follow the $1^{\text {st }}$ order Markov assumption, i.e., the future $\boldsymbol{X}^{t+1}$ is independent on the past $\boldsymbol{X}^{t-1}$ given the present $\boldsymbol{X}^{t}$ : $\left(X^{t+1} \perp X^{t-1} \mid X^{t}\right)$ together with the stationary assumption, that the transitional probability distributions do not change between the time slices:

$$
P\left(X^{t+1} \mid X^{t}\right)=P\left(X^{t} \mid X^{t-1}\right) .
$$

\section{OOBN for Maneuver Recognition}

To recognize the maneuvers considering the relative vehicle-vehicle motion (Figure 2), we have modeled them as states of variable MNVR(=Maneuver of Pairs) 
at the top layer (Figure 3, Figure 4) of the OOBN. The pairwise combination of vehicles' maneuvers ensures scalability of the approach. It reduces the memory requirements and uses computation resources only for the actually present surrounding vehicles. The OOBN fuses in the hypotheses at the lowest level of abstraction (see Figure 3) features under uncertainties (Figure 5, Figure 6), i.e. measured multi-sensor data and computed situation features.

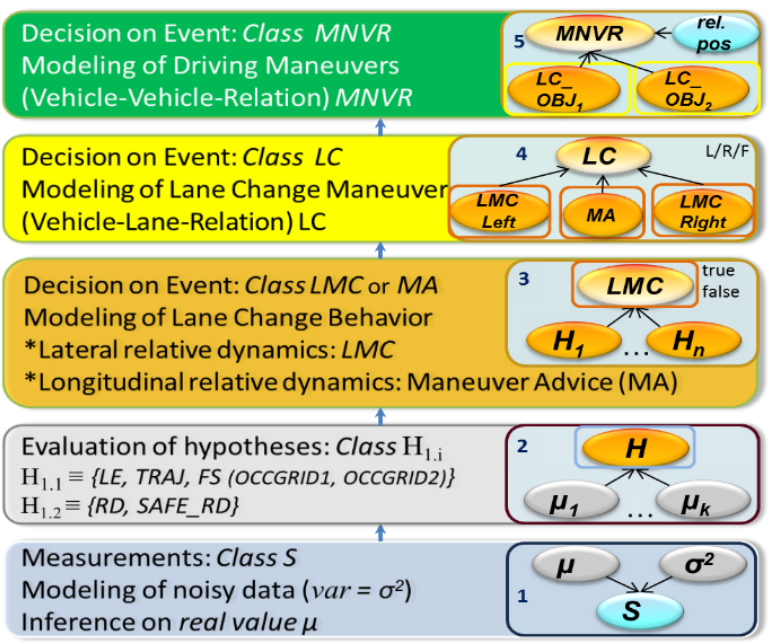

Figure 3. OOBN structure and layers for maneuver recognition.

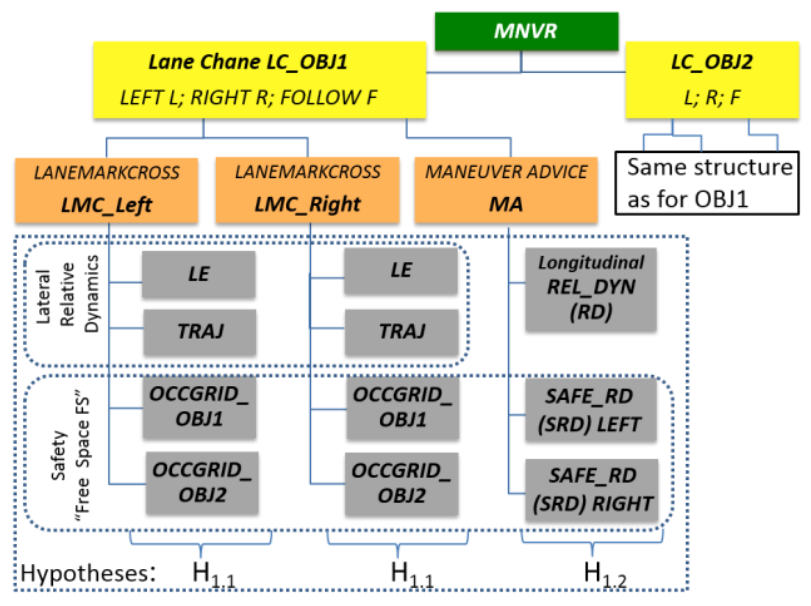

Figure 4. Class hierarchy of the OOBN for maneuver recognition between two vehicles (OBJ1 and OBJ2), expressed as lateral and longitudinal relative dynamics, including their safety (see Figure 1).

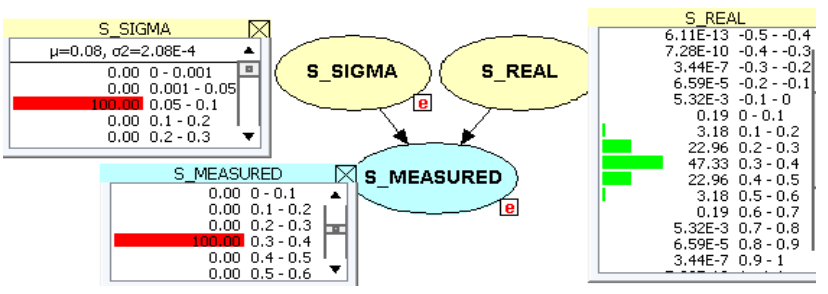

Figure 5. BN fragment for modeling of sensor's uncertainties with a discrete MEASURED variable.

\section{Modeling of Data and Driving Behavior}

\subsection{Modeling of sensor data and its uncertainty}

The maneuver recognition represents a task of the type reasoning under uncertainties with heterogeneous data. These data are acquired from multi-sensors measurements as well as from thereof fused and computed (by physical models) situation features. All data have naturally inherited uncertainties. The data characterize a traffic situation and define the set of situation features for maneuver recognition. The data input is represented as variables in the OOBN to support the inference process by allowing the measured (or computed) values of the variables to be inserted as evidence. For discrete variables, to be able to distinguish between the states of deduced features and to deal with the uncertainties in the sensor signals (Kaper et al, 2011, 2012,2013 ), the measured signals are discretized in predefined partitions (Figure 5). In general, the measured signal $S_{\text {measured }} \equiv S_{m}$ is composed of its real (expected) value S_REAL $\equiv S_{\text {expected }}$ under measurement and its disturbance (sensor noise) $S_{e r r}$ around the real value, i.e. $S_{m}=S_{\text {expected }}+S_{\text {err }}$. In many practical applications (and in our work), the sensor noise is assumed as a zero-mean Gaussian random process. Then, the disturbance is described by the signal variance $S_{\text {err }} \equiv S_{\sigma}^{2}$.

If the measurement instrument is not functioning properly (due to senor noise or fault), then the sensorreading $\left(\mathrm{S}\right.$ MEASURED $\equiv S_{m}$ ) and the real variable (S_REAL) under measurement do not need to be the same. This fact imposes the causal model structure as shown in Figure 5, taking care of the uncertainties in the input data. The sensor-reading $S_{m}$ of any measured variable is conditionally dependent on random changes in two variables: real value under measurement $\left(\mathrm{S} \_\mathrm{REAL} \equiv S_{\mu}\right)$ and sensor fault $\left(\mathrm{S} \_\mathrm{SIGMA} \equiv S_{\sigma}^{2}\right)$ :

$$
P\left(S_{m} \mid S_{\mu}, S_{\sigma}^{2}\right)=N\left(S_{\mu}, S_{\sigma}^{2}\right)
$$

where $\mathrm{N}\left(S_{\mu}, S_{\sigma}^{2}\right)$ denotes the Gaussian distribution with mean value $\mathrm{S} \_$REAL $\equiv S_{\mu}$. Then, in principle, the probability distribution of the real value $S_{\mu}$ of the measured variable is inferred by equations (2) - (3), given the observation (evidence) from its sensor measurement $S_{m}$ and its sensor disturbance $S_{\sigma}^{2}$. The last is obtained from the sensor diagnostics by use of a Kalman filter.

In the discrete case, the CPD of $S_{m}$, expressed as (3), is represented by a conditional probability table (CPT), while in the continuous case $S_{m}$ is modeled by a continuous random variable with a linear continuous Gaussian (CG) conditional distribution function $N\left(S_{\mu}\right.$, $S_{\sigma}{ }^{2}$ ). A BN with CG nodes is referred to as a Conditional 
Linear Gaussian BN. It induces a multivariate normal mixture density of the form:

$$
P(\Delta) f(\Gamma)=\Pi_{X \in \Delta} P(X \mid p a(X)) \Pi_{X \in \Gamma} f(X \mid p a(X)),
$$

where $\Delta$ are the discrete and $\Gamma$ are the continuous variables.

The degree of uncertainty for a variable, which is computed from noisy sensor data measurements, is obtained as error estimation by variance calculus. For example, the uncertainty in velocity $\Delta v$ and in distance measurement $\Delta s$ directly affects the uncertainty of the computed time to reach a certain point at distance $s$ with velocity $v$. The time is computed as a function of these two variables, i.e. $t=\frac{s}{v}=f(s, v)$. Its uncertainty value or its variance $t_{\sigma}^{2} \equiv \delta t$ is computed for each vehicle object by taking the partial derivative of the time function $f(s, v)$ :

$$
\begin{array}{r}
\delta t=\sqrt{\left(\frac{\delta f}{\delta s}\right)^{2}+\left(\frac{\delta f}{\delta v}\right)^{2}}=\sqrt{\left(\frac{\partial f}{\partial s} \delta s\right)^{2}+\left(\frac{\partial f}{\partial v} \delta v\right)^{2}} \\
=\sqrt{\left(\frac{1}{v} \cdot \Delta s\right)^{2}+\left(-\frac{s}{v^{2}} \cdot \Delta v\right)^{2}}
\end{array}
$$

To model the uncertainty in a variable, which has been computed from noisy sensor measurements, we use Normal (continuous linear Gaussian LCG) distribution. By analogy to (3), similar distribution holds for its conditional probability, which is expressed by (4)

$$
P\left(t_{\text {computed }} \mid t_{\mu}, t_{\sigma}^{2}\right)=N\left(t_{\mu}, t_{\sigma}^{2}\right)
$$

and its causal model structure is shown in Figure 6. Here, the computed variable $t_{\text {computed }}$ is denoted by an ellipse with a double line boarder. The root nodes - real value denoted as $t_{-}$real $\equiv t_{\mu}$ and the sensor noise denoted as $t \_$sigma $\equiv t_{\sigma}{ }^{2}$ - are modeled as discrete variables with uniform distributions for the purpose of independent treatment of the influence variables on the computed variable.

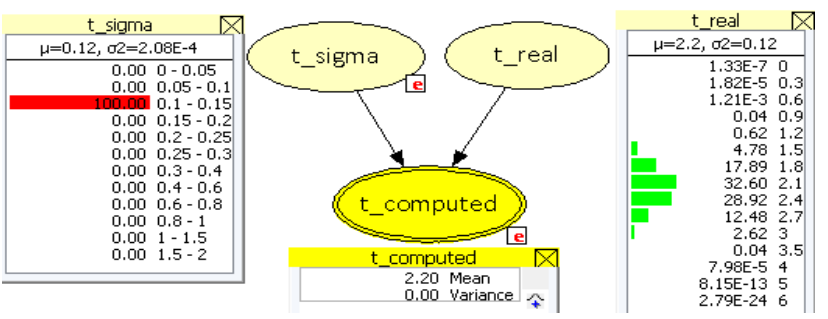

Figure 6. BN fragment, modeling uncertainties in a continuous variable $t_{\text {computed }}$, influenced by discrete variables $t_{\mu}$ and $t_{\sigma}^{2}$.

Similar principle of error estimation can be applied to any computed variable of interest for the BN modelling.
The computed uncertainties complete the set of situation features, used for maneuver recognition.

In (Weidl et al, 2014), we proposed a number of modelling approaches to meet the automotive requirements on RAM and ROM memory size, and on computation time. These included besides the continuous nodes, also the use of function nodes as an alternative modeling of the sigmoid growth of probability for the hypotheses nodes; the use of expressions to specify the conditional probability distributions (CPDs) compactly and a divide-andconquer approach to update of probability.

\subsection{Modeling of driving behavior (hypotheses)}

The lateral relative dynamics (Figure 4) is inferred from the actual lateral movement of a vehicle towards the lane marking. It is fused from the set of hypotheses $\mathrm{H}_{1.1} \equiv\{$ lateral evidence LE, actual movement trajectory TRAJ and free space FS, computed by the occupancy grid OCCGRID\}. Here, the hypothesis LE fuses the vehicle's lateral offset to the lane marking (O_LAT) and its lateral speed (V_LAT) as shown in Figure 7. Its CPD is represented by a sigmoid function to expresses the growing probability for LE (and possible lane change) when the vehicle is coming closer to the lane marking (modeled by O_LAT_MEASURED) by growing lateral velocity (modeled by V_LAT_ MEASURED (see (Kaper et al, 2011, 2012, 2013) and for modeling optimization with continuous variables - see (Weidl et $a l, 2014)$. By analogy, the hypotheses TRAJ fuses: lateral acceleration (A_LAT), gear angle (vehicle's orientation in the lane) and the time-to-lane-crossing. For safety of lane change, $\mathrm{H}_{1.1}$ checks available free space by assessing the risk of simultaneous occupancy of surrounding target cells (OCCGRID). This free space is inferred based on the times to enter and to leave the occupancy cells (Kaper et al, 2011, 2012, 2013).

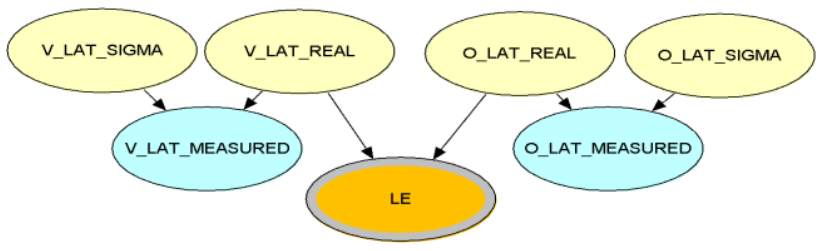

Figure 7. BN fragment modeling the hypothesis LE with discrete variables V_LAT_MEASURED and O_LAT_MEASURED.

The longitudinal relative dynamics (Figure 1, Figure 4) is fused from the set of hypotheses $\mathrm{H}_{1.2} \equiv$ \{longitudinal relative dynamics (RD) and its safety SAFE_RD \}, see section 3. In the Original OOBN the BN-hypothesis $L E$, used for the evaluation of $L M C$ (Figure 3, Figure 4), can recognize a maneuver only when the car approaches the lane marking. Hence, the intention of a driver to make a lane change cannot be detected with it. Therefore, we explore the longitudinal 
relative dynamics (Figure 1) which is characterized by hypothesis "Relative Dynamics" $\left(R E L \_D Y N \equiv \mathrm{RD}\right)$. Here we use the radar-measured features, characterizing the relation between a follower-vehicle and its frontvehicle on the same lane. The radar provides additional advantage of a longer view-horizon (up to $200 \mathrm{~m}$ ) than the camera (up to $60 \mathrm{~m}$ ). Since the hypotheses $R E L \_D Y N$ is contributing to the recognition of maneuver intention, it can be considered as "Maneuver Advice" and should be integrated in the higher abstraction OOBN layers, i.e. into the third layer in parallel with LMC and its output on "Maneuver Advice" further into the forth layer LC of the OOBN (Figure 3, Figure 4), since we use information on how fast the vehicles in front on the same lane are driving.

First, we apply the model for handling of uncertainties in measurements (described in section 4.1) - see layer 1, Figure 3. For simplicity, we will take two measured features (relative distance $X \_R E L \_M E A S$ and relative velocity $V_{-} R E L \_M E A S$ to the vehicle in front) and their variances $\sigma^{2}$ to improve the maneuver recognition time performance, i.e. earlier as with hypotheses $\mathrm{H}_{1.1}$. The structure of the static BN-Model on relative dynamics is shown in Figure 8.

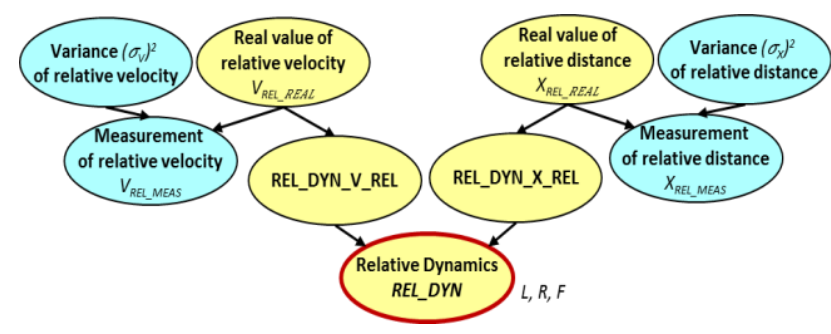

Figure 8. Static BN-Model for hypothesis "Relative Dynamics". Evidence nodes are coded with blue color; chance nodes - with yellow; decision hypothesis for maneuver $\mathrm{L} / \mathrm{R} / \mathrm{F}$ - with red border.

At the next abstraction level: $\mathrm{H}_{1.1}$ contribute to the recognition of an event lane marking crossing by class LMC $\equiv$ LANEMARKCROSS (where the classification node LMC is Boolean), which is reused at the next abstraction level for the recognition of LEFT and RIGHT lane marking crossing to infer on event $\mathrm{LC} \equiv$ lane change for each vehicle (OBJ1 and OBJ2). The OOBN model structure and parameters are based on domain knowledge and physical models, as described in details in (Kaper et al, 2011, 2012, 2013; Weidl et al, 2014, 2015). The initial OOBN parameterization has been specified qualitatively and quantitatively by use of expressions, like sigmoid functions or kinematics relations. The parameters have been initially hand tuned by domain experts to reflect expected lane change behavior for the conditional probability distributions of the hypotheses variables, which represent qualitatively a typical vehicle behavior at lateral and longitudinal relative dynamics with safety aspects.

\subsection{Free Space Model for Safety}

The CUTOUT and CUTIN maneuvers (Figure 1, Figure 2) can be considered as a lateral relative dynamics motion, since they represent a vehicle, performing a lateral movement towards the lane marking and relative to neighbor vehicles. In addition to the lateral relative dynamics, the longitudinal relative dynamics becomes essential for earlier recognition of maneuver intentions on lane change. It assumes, the analyzed vehicle aims to keep certain comfortable speed during its highway drive. It considers the longitudinal relative speed and relative distance to a vehicle driving in front on the same lane, Figure 1. The modeling principle of safety for the longitudinal relative dynamics is similar to the safety for the lateral dynamics, relative to the lane marking crossing (LMC), i.e., the hypothesis lateral Free Space (FS) in (Kasper, 2013). For safety, the longitudinal relative dynamics requires the check of available free space on the target lane to finish a maneuver or the suitability of a gap between two neighbor vehicles. This is performed by evaluating the safety features for longitudinal relative dynamics: "SAFE_RD/LEFT or RIGHT" (Figure 4). The driving praxis shows, that if a vehicle in front is slower, usually it is overtaken on the left, if free space is available (Figure 1). On the other hand, when a vehicle intends to leave the faster moving lane, it is slowing to change to the most-right or to the highway exit lane. Therefore, to ensure safety, it is necessary to estimate two features: the relative velocity and time to collision with vehicles on the target lane. These features are calculated as a relation to the nearest vehicles on the target lane, both behind and in front of the analyzed vehicle with a possible intention of a lane change. This safety mechanism is reflected in the fusion of the mentioned features, which are calculated for the left and right neighbor lanes. Figure 9 shows the BN fragment structure of the hypothesis "Safe_RD" for longitudinal relative dynamics. By analogy to the evaluation of the longitudinal relative dynamics to the front vehicle on the same lane (Tereshchenko, 2014; Weidl et al, 2015), a similar structure is used to model the relation to both the front and behind moving vehicle on the target lane (same fragment is used to evaluate both its left and right side).

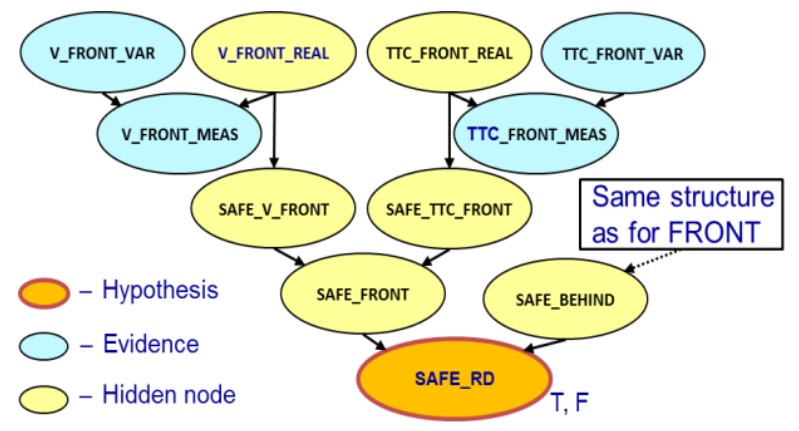

Figure 9. Safe hypothesis (SRD) for longitudinal Relative Dynamics. 
The "Safe_RD" output nodes represent the interface nodes at the next layer of abstraction (Maneuver Advice $\equiv$ MA, Figure 4).The evidence features are modeling the measurements (denoted as *_MEAS) with their uncertainty (variance denoted as *_VAR) which are assumed to have a Gaussian distribution. The distributions of nodes (V_FRONT_REAL, TTC_FRONT_REAL, etc.) are inferred based on the evidence. Nodes SAFE_V_FRONT and SAFE_TTC_FRONT are fused as an OR-relation in node SAFE_FRONT, i.e., a lane change to the target lane is safe only if at least one of the nodes has high probability. Thus, it models the relation between the "FRONT" input variables and the safety ahead on the neighbor target lane of the considered front vehicle. The CPD of SAFE_BEHIND is parameterized by analogy to SAFE_FRONT. Node SAFE_RD (evaluating the gap) combines the results from SAFE_FRONT and SAFE_BEHIND and is implemented as an "AND-relation", i.e. if both have a high probability for state "true", SAFE_RD will have also high probability for "true". However, if one of them is in state "false", SAFE_RD will have a high probability for "false".

\subsection{Dynamic Models for Earlier Recognition}

Here, we focus on the use of two-time slice dynamic Bayesian networks DBNs (2T-DBNs) to achieve earlier recognition of traffic maneuvers, see (Weidl et al, 2015). They are characterized by an initial model representing the initial joint distribution of the process and a transition probability distribution (TPD) representing a standard $\mathrm{BN}$ repeated over time. They satisfy both the first-order Markov assumption and the stationary assumption. Figure 10 shows the graphical structure of a $2 \mathrm{~T}-\mathrm{DBN}$ model for the hypothesis LE, while Figure 11 represents a DBN extension of hypothesis REL_DYN, with the hidden node $A_{\text {REL_REAL }}(t)$, which was added for purposes as explained below. The TPDs between the time slices $\mathrm{t}$ and $\mathrm{t}+1$ are assumed conditional Gaussian $N\left(\mu, \sigma^{2}\right)$. Here, since we do not have observations on the mean value $\mu$, it is specified by physical models.

$L E \_D B N$ (Figure 10) is combining the real values of three lateral features: $O_{L A T_{-} R E A L}(t), V_{L A T_{-} R E A L}(t)$ and $A_{L A T_{-} R E A L}(t)$. When $O_{L A T_{-} R E A L}(t)$ is steadily increasing and $V_{L A T_{-} R E A L}(t)$ is high or increasing (requiring also $\left.A_{\angle A T \_R E A L}(t)\right)$, their combination clearly indicates that the vehicle is leaving its lane. Note, that in (Kaper et al, 2011, 2012), ALAT_REAL was included in hypothesis Trajectory (TRAJ) and not as part of $L E$. The TPDs for the LE-variables: $O_{L A T_{-} R E A L}(t), \quad V_{L A T_{-} R E A L}(t)$ and $A_{L A T_{-} R E A L}$ are defined as shown in (5)-(7):

$$
\begin{aligned}
& O_{L A T_{-} R E A L}(t) \sim N\left(O_{L A T_{-} R E A L}(t-1)+V_{L A T_{-} R E A L}(t-1) \cdot \Delta t, \sigma_{O_{-} L A T(t)^{2}}\right) \\
& V_{L A T} \text { REAL }(t) \sim N\left(V_{L A T} \text { REALL }(t-1)+A_{L A T_{-} R E A L}(t-1) \cdot \Delta t, \sigma_{V_{-} L A T(t)^{2}}\right) \\
& A_{L A T_{-} R E A L}(t) \sim N\left(A_{L A T} \text { REAL }(t-1), \sigma_{A_{-} L A T}(t)^{2}\right)
\end{aligned}
$$

The time step $\Delta t$ is the cycle time, i.e., 42 or 60 milliseconds depending on the camera used. The variances $\sigma^{2}$ are modeling the uncertainties of the variables. This dynamic extension incorporates the trend of real values, while their physics relations are represented as causal dependencies between time steps $\Delta \mathrm{t}$. By analogy are defined the TPDs for the REL_DYN features: distance $X_{R E L_{-} R E A L}(t)$ and velocity $V_{R E L_{-} R E A L}(t)$ and the hidden variable relative acceleration $A_{\text {REL_REALL }}(t)$.

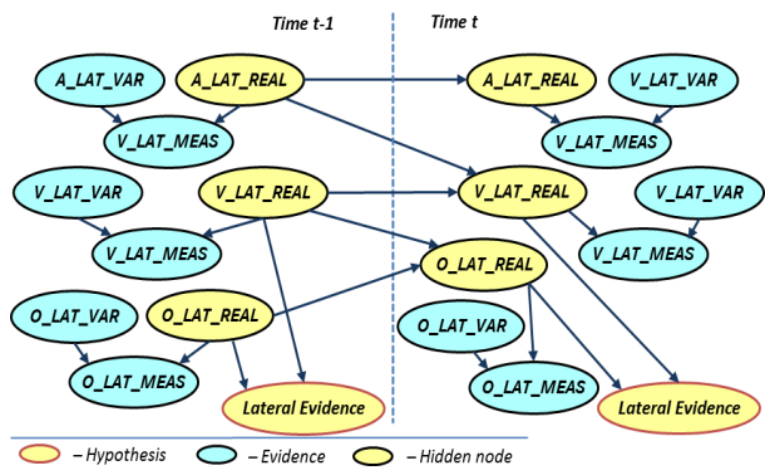

Figure 10. $L E \_D B N$ : $2 \mathrm{~T}$-DBN structure for the hypothesis $L E$ (Lateral Evidence) for lateral Relative Dynamics towards the lane marking.

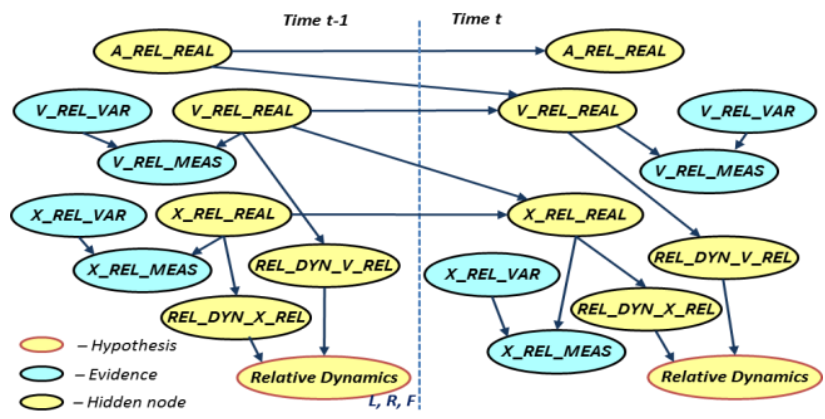

Figure 11. $R E L \_D Y N \_D B N$ : The 2T-DBN structure for the hypothesis $R E L \_D Y N$ (Relative Dynamics) with $A \_R E L \_R E A L$ as hidden node.

\section{Approach}

\subsection{Combination of Methods}

Our approach combines several methods to meet the deployment requirements on accuracy, less memory and faster inference. For resolving of programming paradigms, like efficient modelling and reuse of modeling fragments, we use OOBNs for information fusion from dynamic and/or static fragments. The accuracy requirement is reached by adding "special evidence" as described in section 5.3. Future study will also focus on parameters learning to further improve accuracy. In addition, to resolve the design paradigms for deployment on a prototype vehicle, i.e. to meet the requirements on computation time and memory, we have utilized parallelization of computations based on a divide-and-conquer strategy (D\&C) (Weidl et al, 2014, 
2015, 2017). This D\&C parallelization splits the OOBN model into fragments and uses the posterior distribution of output nodes from the lower hierarchical fragments as likelihood over the corresponding input node at the next level of OOBN hierarchy, see (Weidl et al, 2017) for more details.

\subsection{Modeling of the logical OOBN layers: LMC, MA, Lane Change Maneuvers and Driving Maneuvers (MNVR)}

The network fragments, created to support the divideand-conquer strategy to probability update in OOBN (Figure 3, Figure 4) are shown in Figure 12 and Figure 13. In Figure 12.A), LANEMARKCROSS (LMC) is the object class for lateral relative dynamics towards the lane marking. LMC represents the vehicle-to-lanemarking relation and is instantiated using the probabilities computed in the hypotheses TRAJ, LE, FS (OCCGRID for OBJ1 and OBJ2). In Figure 12.B), Maneuver Advice (MA) is the object class fusing the longitudinal relative dynamics REL_DYN (RD) between two vehicles driving on the same lane, and for safety evaluation on the left or right neighbor lanes, the available free space (Safe_RD) to a front and back vehicles, building the gap for completing a lane change (Figure 1). MA is instantiated using their probabilities.

The event class LANECHANGE (LC) is recognized by fusing LMC and MA (Figure 13). LC is instantiated by the probabilities, obtained from the hypothesis classes LMC towards left and right and from hypothesis class MA. The event class MNVR represents the vehicle-vehicle-vehicle LC-relation (denoted QMVT with 9 states, from all possible Left/Right/Follow LCcombinations of two objects) together with their relative lane-position to each other (denoted as POSDESCR with states: left, right, front). It infers the recognition of predicted maneuver, after instantiation by the probabilities, obtained from the two object classes LC.

\subsection{Improving Accuracy by Special Evidence}

Based on a performance analysis on sequences not included in the evaluation reported below, we have extended the OOBN models with measured/perceived variables representing Special Evidence. The evaluation has been performed with our statistical module and by additional visual examination. As typical for statistical classification, we use a confusion matrix to evaluate the classification results at each time step for all maneuver sequences. The corresponding maneuver state is classified (at each time step) as recognized (i.e. true positive TP, if corresponding to its reference data label) when its probability is bigger than $65 \%$. This threshold value has been empirically derived in (Kasper, 2013). It has been derived from the confusion matrix and from a statistical evaluation of the probability of "false positives" for the Original OOBN on sequences not included in the evaluation reported below. Recall that all six maneuvers (Figure 2) are represented as a state of the MNVR variable, which has moreover one additional state for "DONTCARE" (i.e. pairs without any collision hazard).
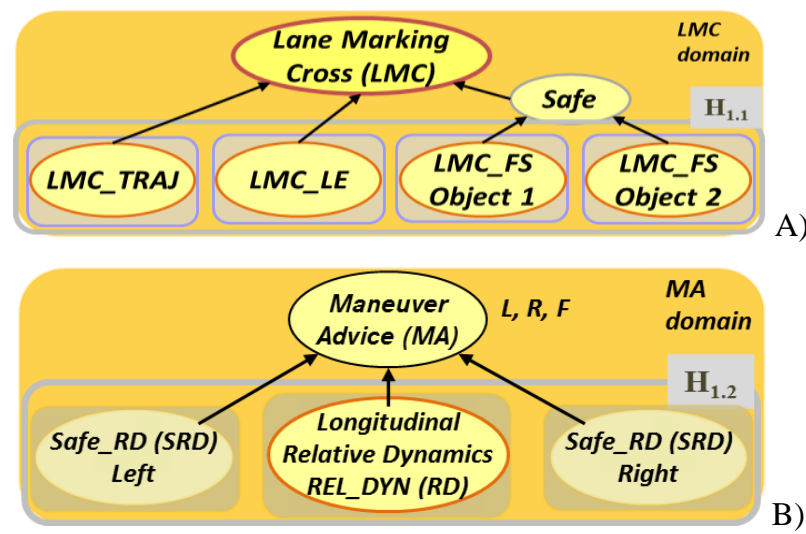

Figure 12. Classes: LANEMARKCROSS (LMC) and Maneuver Advice (MA).

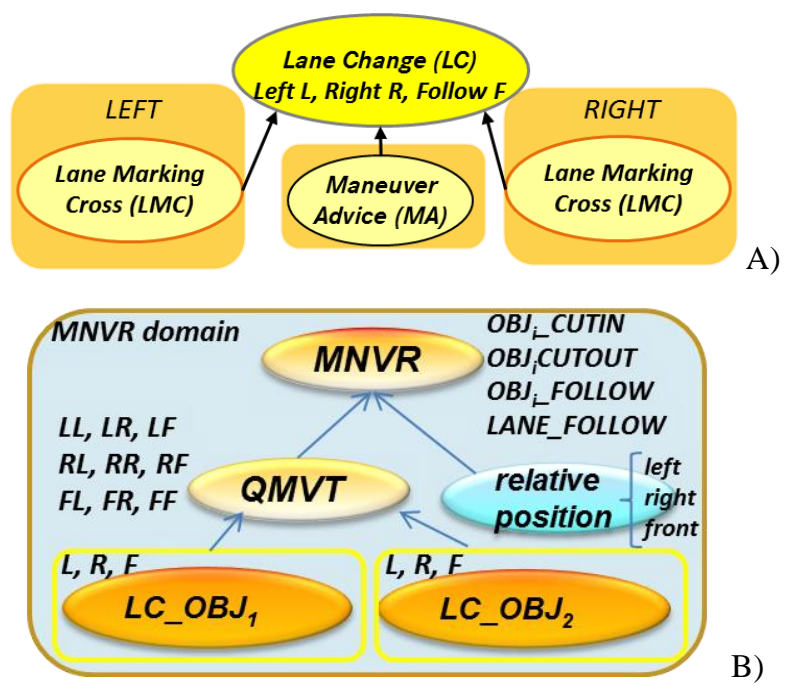

Figure 13. The object classes: LANECHANGE and Maneuver (denoted as MNVR)

We analyzed the results of the statisticsl evaluation and grouped the faults, based on causes with special attention to wrong classifications; and derived ideas for improvement of recognition performance for LaneFollow and Object-Follow situations. We identified some measured/percepted features as "special evidence $\left(S E_{i}\right)$ ", which represent road topology and driving behavior in relation to other vehicles (which are present or not) on the same lane. These $S E_{i}$ features extend the model of Figure 13.B) and are modeled with five blue nodes, while their influence on LC - with the yellow nodes, see Figure 14. Thus, they fuse the information for recognition of a lane change, where SE1 and SE2 consider the lateral and longitudinal relation between each pair of vehicles. 


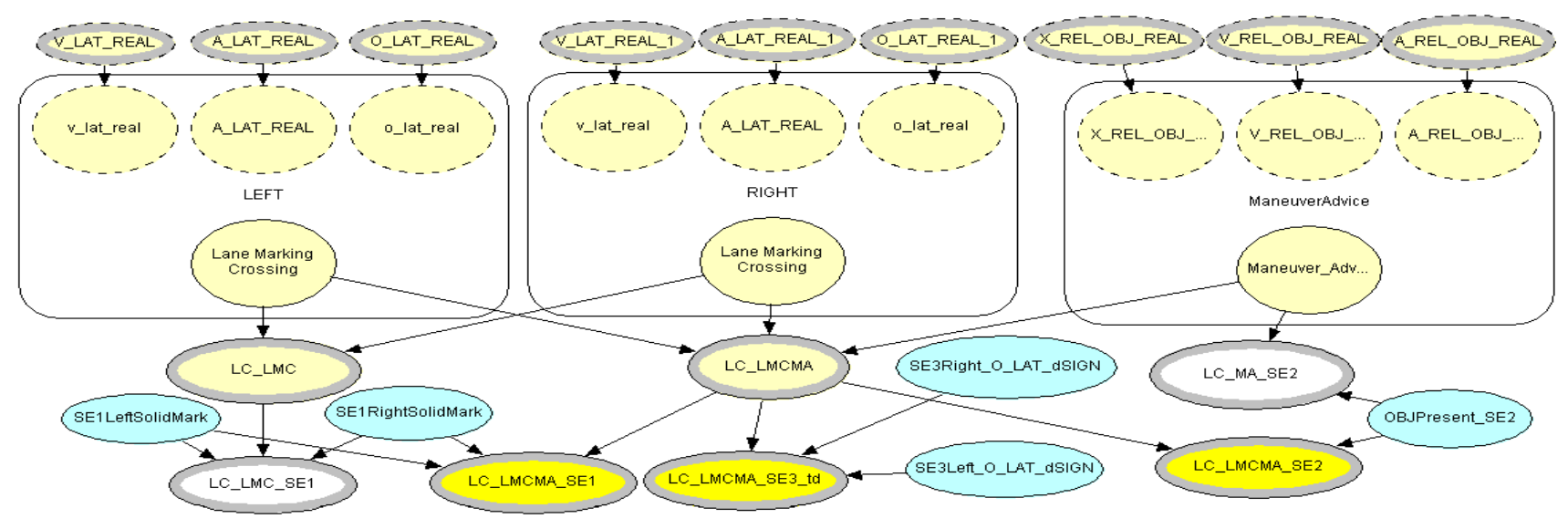

Figure 14. Information fusion at level LC of the extended OOBN model, including "special evidence" nodes to reduce false positive.

SE1: If one vehicle is approaching another vehicle moving on the most left (or most-right) lane, then no matter, if the longitudinal dynamics suggest LC to the left (or right) lane, this is not realizable due to natural lane boarders, i.e., there is no free space to execute any LC LEFT (or RIGHT) maneuver. This is incorporated in the road topology features solid lane markings: SE1_LeftSolidMark, SE1_RightSolidMark. It reduces the number of false positives (FP/wrongly classified) for OBJCUTOUT and the number of false negative (FN/not recognized) for FOLLOW maneuvers by $36 \%$ (from 11 to 7) even in the ORIG model.

SE2: If a vehicle is driving without any vehicle in front of it (possible even due to reduced perception reliability or out of sensor reach), then it has no reason to change the lane, unless an obstacle has been detected. This is incorporated in the model (Figure 14) by SE2 OBJPresent (yes/no). This is represented by the logic rule: If No Front Car detected, then both lateral and longitudinal dynamics classes (Figure 4) - LMC (LaneMarkCross) and REL_DYN - are set to Follow.

SE3: Change of variable sign for lateral offset O_LAT. Due to the used coordinate system, the values of variables are positive only inside the current driving lane and change to negative, while changing to an adjacent lane. This is incorporated as features SE3Right_O_LAT_dSIGN and SE3Left_O_LAT_dSIGN in the extended structure of the BNs, Figure 14. The special evidence is present in the labeled data file and introduced in the models at the LC (Figure 14) and similarly at the maneuver MNVR (Figure 4) level.

\subsection{Deployed classifiers}

To study the effect of different model configurations on recognition, we have defined three static classifiers (ORIG; STATTR; STAT) and one dynamic classifier DBN; see Table 1 . The ORIG classifier uses the Original OOBN (see Kaper et al, 2011, 2012; Kasper, 2013). "Y" shows which BN fragment is included in the corresponding classifier. All static classifiers use hypotheses LE and OCCGRID, while only ORIG and STATTR use TRAJ (Figure 4). The hypotheses "free space" $F S=\{$ OCCGRID and SRD $\}$ for the lateral and longitudinal relative dynamic respectively (Figure 4) remain static $\mathrm{BN}$ fragments for the purpose of satisfying the requirements on computation time and memory. The DBN fragments for the relative dynamics for the lateral and longitudinal motion are LE_DBN and REL_DYN_DBN. The developed static and DBN modelling fragments were generated as c-code and deployed by use of the divide-and-conquer (D\&C) approach for probability update on the target Linux platform of the car - for details see (Weidl et al, 2017).

Table 1. Deployed classifiers on the Linux platform, see Figure 4.

\begin{tabular}{|c|c|c|c|c|c|}
\hline \multirow[b]{2}{*}{ Classifier } & \multicolumn{5}{|c|}{$\mathrm{BN}$ fragment } \\
\hline & $\begin{array}{l}\text { LE } \\
\text { (lateral } \\
\text { relative } \\
\text { dynamics) }\end{array}$ & \begin{tabular}{|l} 
TRAJ \\
(trajec \\
tory)
\end{tabular} & $\begin{array}{l}\text { OCCGRID } \\
\text { OBJ1-OBJ2 } \\
\text { (free space) }\end{array}$ & $\begin{array}{l}\text { REL_DYN } \\
\text { (longitudinal } \\
\text { RD=relative } \\
\text { dynamics) }\end{array}$ & $\begin{array}{l}\text { SRD } \\
\text { (free } \\
\text { spacel } \\
\text { safety) }\end{array}$ \\
\hline ORIG & $\mathrm{Y}$ & $\mathrm{Y}$ & Y & - & - \\
\hline STATTR & Y & Y & Y & $\mathrm{Y}$ & Y \\
\hline STAT & Y & - & Y & $\mathrm{Y}$ & Y \\
\hline$D B N$ & LE_DBN & - & Y & RD_DBN & Y \\
\hline
\end{tabular}

\section{Evaluation and Analysis}

Data sets used in the evaluation: The dataset has been acquired while driving in typical highway traffic. The raw data amounts to Terabytes. They are acquired by radar and stereo camera, which are fused to obtain the data objects with their characteristic features. In order to be able to analyze and use these data, they must be cleaned. The data preparation has involved: i) Visual examination of data quality for all collected sequences 
in the prototype vehicle; ii) Statistical evaluation of all wrongly classified as well as not recognized maneuvers; iii) Data labelling and generation. Steps ii) and iii) have been automated. As a result, we have a total of 336 sequences consisting of 236 lane-change sequences and 100 lane-follow sequences. Quality measures of the recognition results have been selected for the performance evaluation of all developed classifiers. They include: the confusion matrix for the relevant (lane change) and irrelevant (follow or not present in the data) maneuvers; precision, recall; time gain for earlier recognition; and runtime performance of all classifiers. The statistical evaluation module establishes how big the time gain is relative to the labelled maneuver. The last is defined by the actual moment of lane marking crossing (LMC) by the midpoint of the car front bumper.

The confusion matrix for all deployed classifiers (Table 1) as performing on all evaluated driving sequences is shown in Table 2. The BN fragment TRAJ increases the accuracy, but the used gear angle is difficult to measure. The modeled special evidence has been successfully tested to contribute with a reduction of false positives (FP), thus increasing the accuracy. This has improved the performance on the Linux platform for deployment on the prototype car. We have made a proof, based on the performance of the ORIG classifier, by using all sequences and the statistical evaluation module, that the recognition accuracy of OOBN is not affected by the D\&C approach and its implementation.

Table 3 shows the time gain for all maneuver classes for vehicle pairs. The average time gain for all deployed classifiers is about 1 second ahead of LMC. Moreover, dependent on the traffic situation and object perception, even earlier maneuver recognition is feasible. 36 of the tested sequences show earlier recognition with time gains of $1.5 \mathrm{~s}-6.72 \mathrm{~s}$ (seconds). Test drives in real highway confirm, that traffic scenarios with "longitudinal relative dynamics" are recognized as a "need for lane change" before a vehicle is initiating a maneuver due to the recognition of a slower moving vehicle in front on the same lane. Therefore, the recognition by DBN classifier (visualized in Figure 1517 with blue arrow) is earlier than the one by ORIG (visualized with red arrow). Figure 15 shows recognition of EGOCUTOUT, where DBN is $3.24 \mathrm{~s}$ earlier than the actual lane marking crossing (LMC) and $2.46 \mathrm{~s}$ earlier than ORIG. The recognition of OBJCUTOUT (Figure 16) is 4.62 s earlier by DBN than LMC and 3.9s earlier than ORIG.

The driving sequence with the best recognition performance is shown in Figure 17, where OBJCUTIN maneuver is recognized by DBN 6.72s earlier than LMC and $5.88 \mathrm{~s}$ earlier than ORIG. The parallel D\&C implementation has been deployed for all classifier alternatives (ORIG, STAT, STATTR, DBN, see Table 1.) on the automotive Linux platform for maneuver recognition. Table 4 shows between $22 \%$ and $40 \%$ gain in runtime performance for all deployed classifiers (using c-code on the Linux platform) due to the parallel D\&C approach with "special evidence SE". A time performance of 1-4 milliseconds (ms) is still far from the target value of 0.15 milliseconds. One should note here, that these numbers are for the Linux prototype platform of the car and thus hardware dependent. The optimization of the parallel D\&C method on a dedicated Linux computer allowed even better results with optimized time performance, coming very close to the initially set requirement of the target platform, see (Weidl et al, 2017).

Table 2. Performance comparison of the deployed classifiers with "special evidence" (index _SE). Column Nr.: 1: TP of OBJCUTIN; 2: TP of OBJ CUTOUT; 3: TP of EGOCUTIN; 4: TP of EGOCUTOUT; 5: TP (=as labeled); 6: FN (not recognized); 7: FP (wrong classified); 8: All $(\mathrm{TP}+\mathrm{FN})$ Maneuvers

\begin{tabular}{|l|l|l|l|l|l|l|l|l|l|l|}
\hline Classifier & 1 & 2 & 3 & 4 & 5 & 6 & 7 & 8 & precision & recall \\
\hline Label* & 29 & 83 & 67 & 57 & 236 & 0 & 0 & 236 & & \\
\hline ORIG & 25 & 82 & 67 & 57 & 231 & 5 & 11 & 236 & $95.5 \%$ & $97.9 \%$ \\
\hline ORIG_SE & 25 & 82 & 67 & 57 & 231 & 5 & 7 & 236 & $97.1 \%$ & $97.9 \%$ \\
\hline DBN_SE & 23 & 80 & 67 & 56 & 226 & 10 & 19 & 236 & $92.2 \%$ & $95.8 \%$ \\
\hline STAT_SE & 23 & 76 & 67 & 56 & 222 & 14 & 13 & 236 & $94.5 \%$ & $94.1 \%$ \\
\hline STATTR_SE & 25 & 82 & 67 & 56 & 230 & 6 & 16 & 236 & $93.5 \%$ & $97.5 \%$ \\
\hline
\end{tabular}

Table 3. Summary of evaluation results for time gain (negative time value means "maneuver prediction before crossing the lane marking") with all data for all deployed classifiers. 1: OBJCUTIN; 2: OBJCUTOUT; 3 : EGOCUTIN; 4: EGOCUTOUT.

\begin{tabular}{|l|l|l|l|l|l|}
\hline dt [s] & 1 & 2 & 3 & 4 & Avg. dt [s] \\
\hline ORIG & -0.9950 & -1.0129 & -1.1461 & -1.1156 & -1.0749 \\
\hline ORIG_SE & -0.9979 & -1.0129 & -1.1936 & -1.1945 & -1.1089 \\
\hline DBN_SE & -0.8943 & -0.9956 & -1.1186 & -1.3184 & -1.1077 \\
\hline STAT_SE & -0.8977 & -1.0161 & -1.1192 & -1.3092 & -1.1089 \\
\hline STATTR_SE & -1.0150 & -1.1246 & -1.1545 & -1.3500 & -1.1763 \\
\hline
\end{tabular}

Table 4. Runtime Performance.

\begin{tabular}{|l|l|l|l|l|}
\hline $\begin{array}{l}\text { Deployed } \\
\text { classifier }\end{array}$ & $\begin{array}{l}\text { Avg. } \\
\text { Runtime } \\
{[\mathrm{ms}]}\end{array}$ & $\begin{array}{l}\text { Deployed } \\
\text { classifier } \\
\text { by parallel D\&C }\end{array}$ & $\begin{array}{l}\text { Avg.Run } \\
\text { time } \\
{[\mathrm{ms}]}\end{array}$ & $\begin{array}{l}\text { Gain with } \\
\text { parallel } \\
\text { D\&C [\%] }\end{array}$ \\
\hline ORIG & 1.5989 & D\&C_ORIG & 1.2508 & $21.8 \%$ \\
\hline ORIG_SE & 1.5989 & D\&C_ORIG_SE & 1.0471 & $34.5 \%$ \\
\cline { 1 - 2 } DBN_SE & 6.7405 & D\&C_DBN_SE & 4.061 & $39.8 \%$ \\
\hline STAT_SE & 1.9734 & D\&C_STAT_SE & 1.4606 & $26 \%$ \\
\hline STATTR_SE & 2.3143 & D\&C_STATTR_SE & 1.5422 & $33.4 \%$ \\
\hline
\end{tabular}



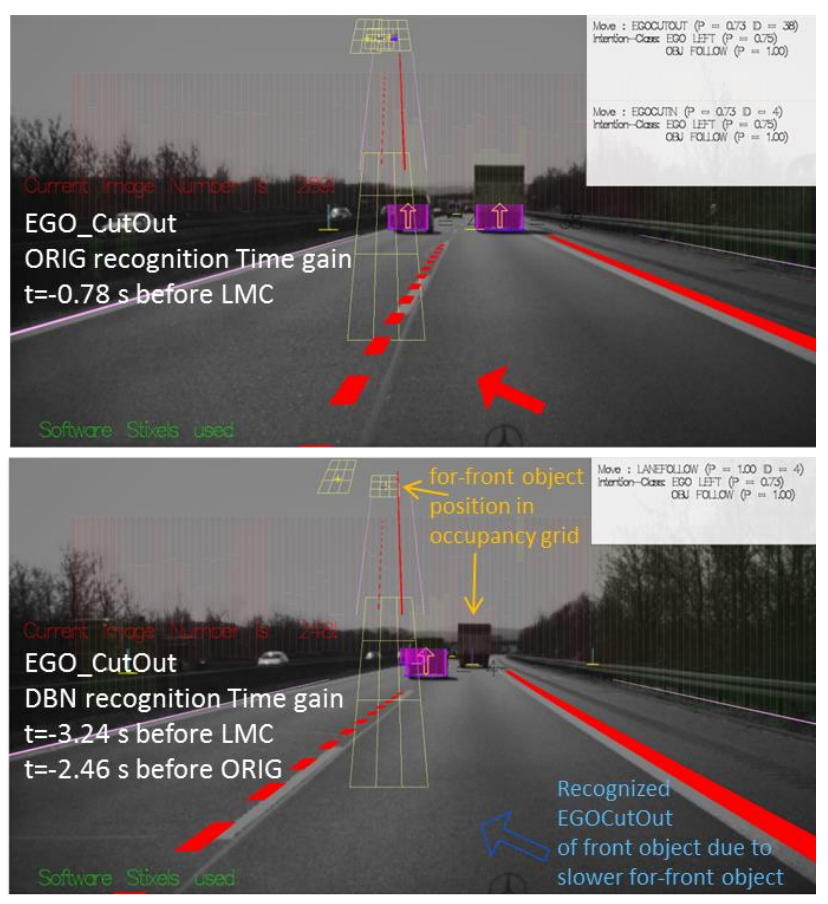

Figure 15. Highway demonstration with REL_DYN showing the classifier performance for EGOCUTOUT: DBN is 3.24 s earlier than actual LaneMarkingCrossing (LMC) and 2.46s - than ORIG.
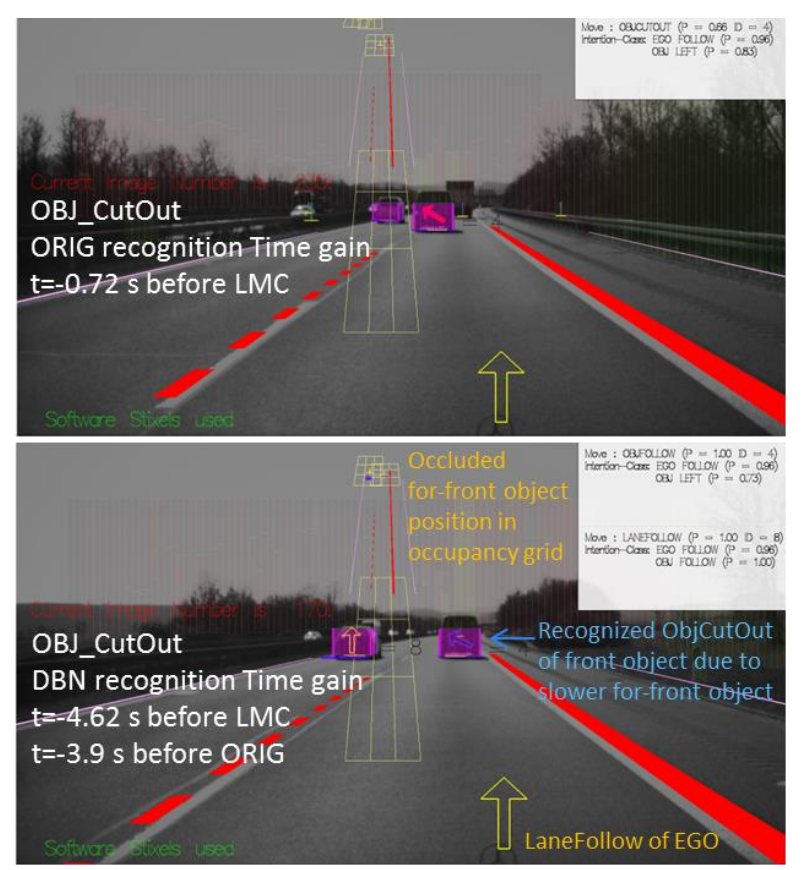

Figure 16. Highway demonstration with REL_DYN showing the classifier performance for OBJCUTOUT: DBN is 4.62 s earlier than actual LMC and 3.9s earlier than ORIG.

\section{Discussion of Results and Outlook}

A Bayesian network has been designed and parameterized for lane change maneuvers. The advantage of our approach is that only measured features for lateral and longitudinal dynamics of the vehicles are necessary, without map data. The limitation is that lane markings are required to compute the features and some wrong classifications cannot be resolved for cases when the prediction horizon of a lane curvature does not reach the percepted front vehicle and thus the vehicle orientation inside the lane cannot be computed.

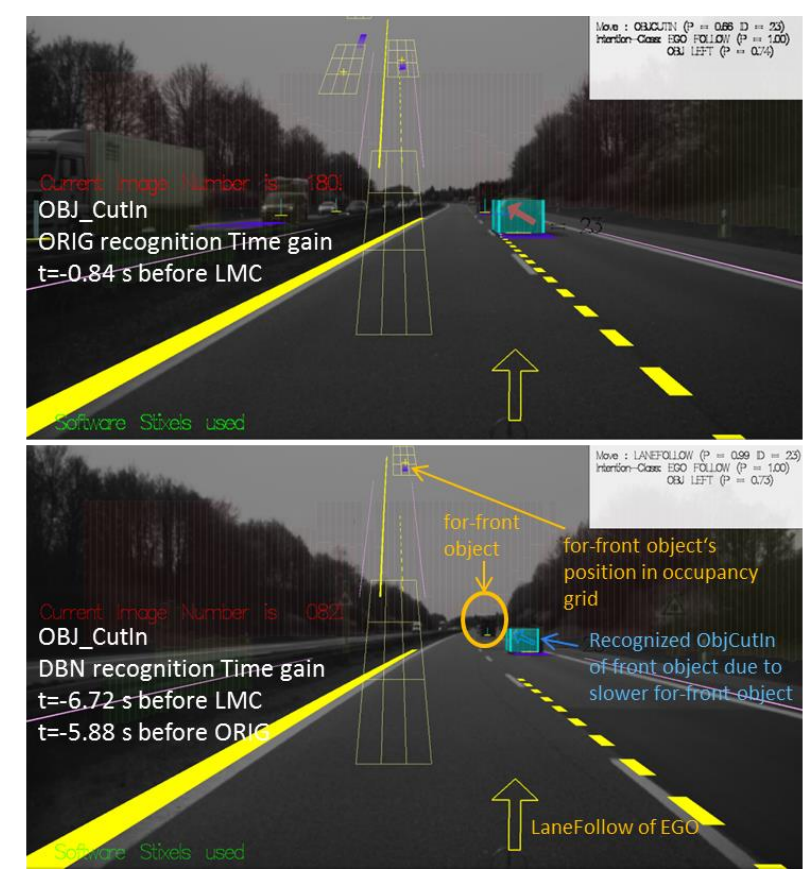

Figure 17. Highway demonstration with REL_D YN showing the classifier performance for OBJCUTIN: DBN is $6.72 \mathrm{~s}$ earlier than actual LMC and $5.88 \mathrm{~s}$ earlier than ORIG.

The introduced "special evidence" reflects road topology and vehicles relations, thus improving the recognition accuracy of lane-follow and reducing the false positives of lane-change maneuvers. The solution has been successfully tested for all classifiers. With the system deployment on the prototype vehicle, we have collected more data, which will be further divided to use for testing, and for learning of models' parameters of the lateral and longitudinal relative dynamics, together with their safety aspects. Here the hand tuned expressions will serve as an initial guess to improve further the recognition accuracy by use of machine learning techniques (Lauritzen, 1995), which have shown promising results.

In addition, we have analyzed the effect of parallelization of computations based on divide-andconquer strategy (D\&C). We describe in (Weidl et al, 2017) the implemented parallel D\&C realization, allowing resolving the requirements on computation time (0.15 milliseconds) and memory for deployment on a prototype vehicle. This is an important step towards a scalable solution, meeting the hardware constraints of the automotive target platform. Future work will also focus on trend analysis for even more accurate and earlier maneuver recognition. 


\section{Acknowledgements}

AMIDST (Analysis of Massive Data Streams) is a project, which has received funding from the European Union's 7th Framework Programme for research, technological development and demonstration under grant agreement no 619209.

\section{References}

N. Friedman and D. Koller. Probabilistic Graphical Models: Principles and Techniques, The MIT Press, 2009. ISBN-13: 978-0262013192.

D. Kasper, G. Weidl, T. Dang, G. Breuel, A. Tamke, and W. Rosenstiel. Object-oriented Bayesian networks for detection of lane change maneuvers. IEEE Intelligent Vehicles Symposium (IV), 2011. doi: 10.1109/IVS.2011.5940468.

D. Kasper. Erkennung von Fahrmanöovern mit objectorientierten Bayes-Netzen in Autobahnszenarien. PhDThesis, Tübingen University Germany, 2013.

D. Kasper, G. Weidl, T. Dang, G. Breuel, A. Tamke, A. Wedel, and W. Rosenstiel. Object-oriented Bayesian networks for detection of lane change maneuvers. IEEE Intelligent Transportation Systems Magazine, vol.4, pp. 1931, 2012. doi: 10.1109/MITS.2012.2203229.

U. B. Kjærulff and A. L. Madsen 2013, Bayesian Networks and Influence Diagrams - A Guide to Construction and Analysis. Springer. Second Edition, 2013. doi: 10.1007/978-1-4614-5104-4.

P. Kumar, M. Perrollaz, S. Lefevre and C. Laugier. LearningBased Approach for Online Lane Change Intention Prediction. IEEE Intelligent Vehicles Symposium (IV), Gold Coast, Australia, 2013.

S. L. Lauritzen. The EM algorithm for graphical association models with missing data. Computational Statistics and Data Analysis, vol. 19, pp. 191-201, 1995. doi: 10.1016/0167-9473(93)E0056-A.

G. Li, S. E Li, Y. Liao, W. Wang, B. Cheng and F. Chen. Lane change maneuver recognition via vehicle state and driver operation signals - Results from naturalistic driving data. IEEE Intelligent Vehicles Symposium (IV), pp.865-870, 2015. doi: 10.1109/IVS.2015.7225793.

A. Locken, H. Muller, W. Heuten and S. Boll. An experiment on ambient light patterns to support lane change decisions. IEEE Intelligent Vehicles Symposium (IV), pp. 505-510, 2015. doi: 10.1109/IVS.2015.7225735.

M. Mori, K. Takenaka, T. Bando, T. Taniguchi, C. Miyajima and K. Takeda. Automatic lane change extraction based on temporal patterns of symbolized driving behavioral data. IEEE Intelligent Vehicles Symposium (IV), pp. 976-981, 2015. doi: 10.1109/IVS.2015.7225811.

B. Morris, D. Anup and T. Mohan. Lane Change Intent Prediction for Driver Assistance: On-Road Design and Evaluation. IEEE Intelligent Vehicles Symposium (IV), vol. IV, pp. 895-901, 2011. doi: 10.1109/IVS.2011.5940538.

R. K. Satzoda, P. Gunaratne and M. M. Trivedi. Drive quality analysis of lane change maneuvers for naturalistic driving studies. IEEE Intelligent Vehicles Symposium (IV), pp. 654659, 2015. doi: 10.1109/IVS.2015.7225759.
J. Schlechtriemen, A. Wedel, J. Hillenbrand, G. Breuel and K. D. Kuhnert. A lane change detection approach using feature ranking with maximized predictive power. IEEE Intelligent Vehicles Symposium (IV), pp. 108-114, 2014. doi: 10.1109/IVS.2014.6856491.

V. Tereshchenko. Relative object-object dynamics for earlier recognition of maneuvers in highway traffic. Master's thesis, IAS, University of Stuttgart, Germany, 30.10.2014.

G. Weidl, A. L. Madsen, D. Kasper and G. Breuel. Optimizing Bayesian networks for recognition of driving maneuvers to meet the automotive requirements. IEEE Multi-Conference on Systems and Control, 2014. doi: 10.1109/ISIC.2014.6967630

G. Weidl, A. L. Madsen, V. Tereshchenko, D. Kasper and G. Breuel. Early Recognition of Maneuvers in Highway Traffic. chapter: Symbolic and Quantitative Approaches to Reasoning with Uncertainty. In Lecture Notes in Computer Science, vol. 9161, pp. 529-540, 2015.

G. Weidl, A. L. Madsen, S. Wang, D. Kasper and M. Karlsen Early and accurate recognition of highway traffic maneuvers considering real world application: a novel framework using Bayesian networks. IEEE Intelligent Transportation Systems Magazine, accepted to appear in 2017.

F. Yan, L. Weber and A. Luedtke. Classifying driver's uncertainty about the distance gap at lane changing for developing trustworthy assistance systems. IEEE Intelligent Vehicles Symposium (IV), pp. 1276-1281, 2015. doi: 10.1109/IVS.2015.7225858.

J. Zeisler, J. Cherepanov and V. Haltakov. A driving path based target object prediction. IEEE Intelligent Vehicles Symposium (IV), pp. 316-321, 2015. doi: 10.1109/IVS.2015.7225705.

Y. Zhang and Q. Ji. Active and dynamic information fusion for multisensor systems with dynamic Bayesian networks. IEEE Transactions on System\&Cybernetics, vol. 36/2, pp. 467-472, 2006. doi: 10.1109/TSMCB.2005.859081. 